\title{
Identités de marques et marqueurs d'identité Vers une construction identitaire et sociale des individus par et dans la consommation?
}

\author{
Anne PARIZOT ${ }^{1}$ \\ Claudine BATAZZI ${ }^{2}$
}

On n'a jamais autant évoqué le concept d'identité que depuis l'avènement de la mondialisation et de la globalisation. Les processus d'internationalisation favorisent paradoxalement l'émergence de nouvelles frontières et le collectif s'efface devant un certain individualisme. Des interrogations naissent parmi lesquelles l'identité semble être au cœur de la réflexion dans le contexte mondial. Les pressions ressenties au sein de la société fragmentent les individus en différents «moi » et l'identité s'en trouve fortement fragilisée.

Ainsi, fleurissent dans pléthore de discours sur la société, des expressions telles «crise identitaire » ou «crispations identitaires » qui reflètent un certain malaise dans la société actuelle. Le concept de quête identitaire rejaillit dans un contexte de désenchantement global comme réponse au besoin de quête existentielle.

La mutation de la consommation liée notamment aux TIC et à la crise économique conduit à un affaiblissement des phénomènes de surconsommation se manifestant par une critique globale du capital, une remise en cause des marques et une perte de confiance générale. Les consommateurs recherchent du sens dans cet univers marqué par la perte de repères familiaux, religieux ou spirituels. Ainsi les marques, nouveaux repères des existences désenchantées, valorisent l'expérience individuelle des consommateurs en se créant des identités en accord avec les valeurs actuelles. Les consommateurs se construisent leur identité dans le collectif des communautés et font de la marque, un repère identitaire.

L'objectif de cette communication tendra donc à préciser comment se manifestent les interactions entre les marques et les consommateurs dans ce nouvel univers de la consommation, profondément empreint d'une quête identitaire.

\section{Les nouveaux visages de la consommation}

La consommation, terme polysémique (consumere, détruire et consummare, mener à son terme) aurait causé le désenchantement de la société moderne (M. Weber). Pour B. Heilbrunn (2010) « la consommation renvoie [...] à un ensemble de pratiques identitaires par lesquelles les individus structurent leur identité sociale par d'incessants mécanismes d'échange ». La consommation actuelle n'est pas restreinte à l'acquisition de produits mais relève plutôt de la signification accordée à ces derniers, la valeur symbolique permettant d'augmenter la valeur économique.

\footnotetext{
${ }^{1}$ Professeure de sciences de l'information et de la communication à l'Université de Bourgogne Franche-Comté (Besançon).

${ }^{2}$ Professeure de sciences de l'information et de la communication à l'Université Nice Côte d'azur.
} 
La consommation devient une expérience de consommation, élément clé de la compréhension du comportement du consommateur, mis ainsi en scène et théâtralisé (Hetzel, 2002) par le marketing expérientiel visant la quête identitaire du consommateur,

Cette quête de sens, de reconnaissance des individus est une quête identitaire dans la mesure où «les traditions, la religion, le politique sont moins productrices d'identité sociale, (Lipovestsky, 1980).

Lipovetsky (2003) évoque la société d'hyperconsommation des années 1980, alors qu'émerge déjà un nouveau modèle de consommation qui en ferait une démarche d'appropriation symbolique de biens et de services en objets culturels. La satisfaction des besoins se voit détrônée par la recherche de sensations et d'émotions dans la consommation qui participent activement à la construction de l'identité des consommateurs. Les pratiques de consommation évoluent et privilégient l'expérience valorisée par l'esthétisme et le symbolique.

Cette volonté de vivre des expériences de la part du consommateur s'accompagne du développement du marketing tribal, expérientiel où l'émotionnel est interpellé. L'expérientiel fait appel à un vécu personnel chargé en émotions. Le "marketing indiciel identitaire » où « la société est un réservoir d'expériences à vivre et à créer, de sens à extraire, permet aux individus de construire et d'ajuster leur identité » O. Badot (2006).

La consommation fournit un cadre dans lequel l'individu se met en récit. Par ses choix de consommation, il développe une extension de lui-même que les marques doivent prendre en compte. Par la consommation de biens symboliques, il vise l'amélioration de son image et cherche à se construire une identité spécifique. Ainsi les objets tels qu'ils sont consommés actuellement intègrent un système sémiotique et deviennent des supports de communication de la personnalité et de l'appartenance culturelle des individus.

Le domaine économique et marchand est particulièrement impacté par la notion d'identité. Il convient de tenir compte de la relation d'interaction entre la marque et le consommateur. Reprenons dans un premier temps le concept de marque afin de le définir pour envisager ensuite les modes de relations avec l'identité.

\section{Des marques chargées d'émotions}

Les marques ont envahi le marché et caractérisent la société de consommation. Elles sont très présentes quotidiennement et certains leur vouent un véritable culte. Pour les jeunes par exemple, les marques de sport participent à leur mode d'expression et à leur manière d'être. Oxbow ou Quicksilver sont des symboles identitaires qui transcendent de nombreux aspects de la vie des jeunes (Bouchet et Hillairet, 2009).

Les marques, face aux marchés de plus en plus concurrentiels, sont confrontées à la question suivante : comment se distinguer ou se différencier face à l'offre existante et au pouvoir de négociation grandissant des clients-consommateurs?

La marque correspond avant tout à une identité : l'identité visuelle regroupe le nom, le logo, la charte graphique et les couleurs. Mais elle ne peut se réduire à cette seule existence visuelle. L'identité se voit aussi transcendée par un slogan, une signature. Les marques s'apparentent à des mécanismes de manipulation de signifiants et de signifiés qui conduisent à des systèmes de production de sens. 
Le positionnement définit la personnalité par rapport à la concurrence, au marché et à sa catégorie. La promesse est la raison qui motive l'utilisateur à choisir ce produit ou service. Le nom contribue à la compréhension et facilite la communication. Le territoire englobe les signes graphiques (formes, couleurs, typographies, photographies, rédactions, etc.) qui balisent la création lors de l'élaboration des outils de communication et enfin la communication, peut être apparentée au porte-voix de la marque.

La marque est aussi parole verbale ou non verbale, autrement dit une instance énonciatrice. J.-M. Floch (1995) considère la parole comme un acte, par un processus d'efficacité, de performativité de la promesse à l'engagement. Cette médiation entre parole donnée et parole tenue ou effective s'inscrit dans une plus large stratégie de satisfaction de la clientèle et crée une forme d'attachement.

Mais quelles représentations fournir du concept d'identité dans le domaine de la consommation ? E. Goffman livre des interprétations intéressantes sur la mise en scène ou la mise en récit de l'individu dans ses pratiques de consommation. Le sociologue dégage trois axes (soulignés par B. Heilbrunn, 2010), caractérisant l'individu dans ses interactions : le soi perçu (la façon dont l'individu se perçoit), le soi vitrine (la façon dont on se pense perçu par les autres) et enfin le soi idéal (la façon dont on souhaiterait être perçu). Comme l'individu, la marque se met en scène via un jeu d'interactions, mais in fine conserve une identité propre, tout en se distinguant des autres marques par ses valeurs et la dimension symbolique qui en découle.

La marque constitue la somme de trois éléments qui se combinent: l'image, le positionnement et l'identité.

L'image de marque est la représentation, une interprétation faite par le consommateur. Cette opinion réalisée à un moment donné est éphémère. Il s'agit d'un concept de réception alors que l'identité est un concept d'émission qui doit «spécifier le sens, le projet, la conception du soi de la marque $»$, (Kapferer, $1996: 100)$.

Le positionnement correspond aux caractéristiques par rapport à la concurrence pour rester compétitif sur son marché. La marque doit aussi être attentive aux attentes des clients et prendre en compte l'évolution du marché. Par exemple, Castorama se positionne sur l'axe accompagnateur de clients qui ont peur de se lancer dans le bricolage en créant un programme "Lancez-vous », un site d'entraide. Leroy Merlin invite le client à s'organiser et à s'accomplir par la connaissance ${ }^{3}$. Le slogan «Et vos envies prennent vie » s'inscrit dans un processus de performativité, de réalisation de la pensée, de construction de l'identité. Grâce à Leroy Merlin, le client n'est plus un rêveur ou un sujet passif, mais est un bâtisseur de son propre espace, de sa propre vie.

Cette médiation symbolique de l'émotion suscitée à l'identité construite se retrouve dans la communication publicitaire de nombreuses marques commerciales.

Ainsi seul le positionnement distingue les marques de voitures comparables (allemandes et de luxe) : Audi vise le design alors que BMW a choisi la performance, Mercédès se positionne sur le côté bourgeois. Volkswagen ramène le monde entier de l'automobile à sa propre marque par le slogan « Das Auto ». L'emploi de l'allemand nous fait pénétrer dans l'univers sacré de la qualité et de la haute technologie. A partir de l'émotion suscitée par le ton solennel employé dans la prononciation de ces deux mots, un processus de construction identitaire se forge. On suggère au client qu'en achetant une Volkswagen, il rejoint un cercle d'initiés et

\footnotetext{
${ }^{3}$ Les stratégies culturelles selon les trois fonctions. Brand content, 2016
} 
devient lui-même le représentant de la qualité et de la haute technologie dans tout le secteur automobile.

\section{De la notion d'identité...}

Le concept d'identité, est en pleine évolution depuis le $\mathrm{XX}^{\mathrm{e}}$ siècle et plus particulièrement depuis les années 1960 auquel est substitué celui de co-construction de l'identité.

La relation et les interactions entre marque et consommateur, conjuguées à l'avènement des réseaux sociaux conduit à une double construction identitaire, celle de la marque et celle du consommateur qui se rejoignent tout en étant chacune distincte.

Si la conception traditionnelle semblait opposer identité (essence de la marque elle-même telle qu'elle a été pensée) et image de marque (réception de cette identité), cette distinction n'est plus aussi nette du fait de la co-production liée aux consommateurs (notamment par l'usage numérique). Cependant, les analyses confrontant le donné et le perçu sont toujours présentes et aident à la compréhension globale du fonctionnement d'une marque. Par exemple Wanadoo se veut être une marque à la pointe de l'innovation mais est pourtant perçue par les consommateurs comme une marque plutôt grand public et pas forcément innovante.

L'identité, (dont l'origine idem $^{4}$ signifie identique) a suscité de nombreuses recherches au carrefour de plusieurs disciplines des sciences humaines et de gestion. Elle n'est plus à chercher dans le statut social, les institutions ou les traditions (Dufour-Baïdouri, 2013 : 70) mais s'apparente à un processus dynamique qui, selon Goffman (1975), résulte de négociations interactionnelles avec autrui et aboutit à la construction identitaire.

L'identité se définit par un renvoi à l'histoire, aux valeurs développées. Elle peut définir la spécificité de la marque en rappelant la mission ou la promesse engagée. A titre d'exemples, Darty se réfère à la notion de service basé sur la confiance, Danone, à la nutrition saine, Disney, à l'émotion d'un monde magique.

Michon et Stern (1985) ont dégagé l'identité d'une marque par divers éléments : La raison d'être d'un produit affirmée par le slogan «L'Oréal, parce que je le vaux bien »; le métier révélateur de son existence face à la concurrence qui lui confère une certaine légitimité dans un domaine (l'automobile, la beauté, etc.) ; l'expression des valeurs en relation avec l'éthique (Apple et l'esprit libérateur) ; la légende pour se construire une histoire personnelle (Nike et Michael Jordan le basketteur mythique); le caractère qui vise l'image de l'entreprise; les partenaires marquant la visibilité périphérique et enfin le relationnel qui met en valeur l'émotionnel par exemple (Michelin et son Bonhomme empathique).

Lorsqu'on réunit identité et marque, le prisme de Kapferer (1996:106) devient un outil d'analyse incontournable qui rassemble diverses facettes autour de la relation marqueconsommateur et révèle les forces et les faiblesses de la marque.

L'identité de marque offre la possibilité à la marque elle-même de se valoriser et d'être unique sur le marché : le physique, la personnalité, la culture, la relation, le reflet, la mentalisation.

\footnotetext{
${ }^{4} \mathrm{Cnrtl.fr}$
} 


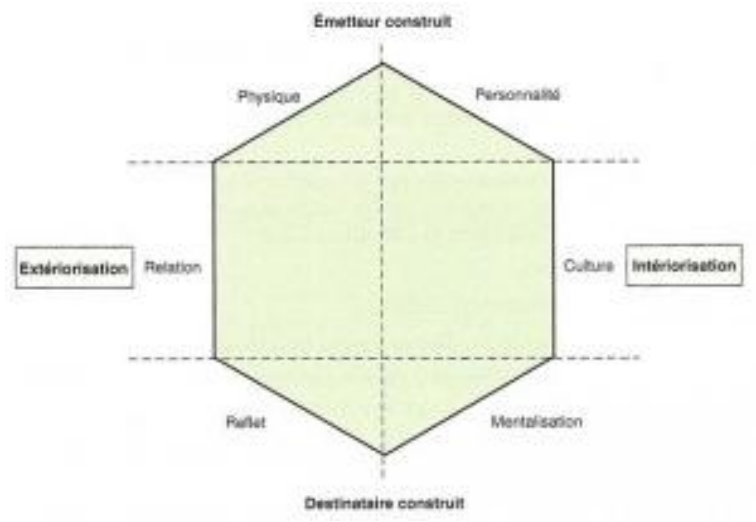

Figure 1 Le prisme de l'Identité Kapferer

La marque est une forme de discours, forme dans le sens de mise en forme, elle correspond à la matérialisation du discours commercial puisque sans communication elle n'existe pas. En ce sens émetteur et récepteur se trouvent construits par et dans cette communication (Ibid. : 112).

Selon F. Berger-Remy ${ }^{5}$,

«Le physique est ce que la marque donne physiquement à voir d'elle-même ; la personnalité fait référence à des traits de caractère attribués par anthropomorphisme. La relation caractérise le type de lien avec les publics. La culture est assimilable à un droit d'inventaire qui précise ce que l'on garde des racines et de l'héritage de la marque. Le reflet et la mentalisation tiennent compte des échanges avec le consommateur. Le reflet est ainsi une description de comment la cible veut être perçue dès lors qu'elle consomme cette marque. On cherche à donner une image de soi par la marque qui nous reflète (Harley: La mentalisation est la réponse à la question «comment je me sens, qu'est-ce que je projette de moi-même du fait de l'utilisation de cette marque $»$.

Pour illustrer chacun de ces éléments, nous pouvons faire référence à des exemples publicitaires.

La marque en tant que forme physique peut être représentée par une calandre (BMW), une bouteille (forme et couleur de la bouteille Perrier), un bonhomme (Michelin). Un lien est alors établi entre le signifiant et le signifié. Et pour rependre le fameux bonhomme en pneus de Michelin (le signifiant), ce dernier nous renvoie à l'assurance (le signifié) d'une bonhommie (entendons par-là confiance et sécurité) dans la conduite.

La personnalité s'illustre par des attributs physiques et psychologiques (Perrier : fou, Ricard : joyeux). La relation se construit à travers le mode de communication et d'échange avec le consommateur. La culture correspond à des valeurs véhiculées par la marque et auxquelles le consommateur aspire (Benetton: la provocation, Apple : Californie). Le consommateur se projette dans la marque qui est mentalisée (Apple : parce que je suis différent).

\footnotetext{
${ }^{5} \mathrm{http}: / /$ chaire.marquesetvaleurs.org/sites/default/files/dt/identite_marque.pdf
} 
F. Berger-Rémy retient également le système d'identité de la marque de D. Aaker centré sur le couple marque - produit. Ce brand identity system définit la marque comme un produit, une organisation, une personne et un symbole.

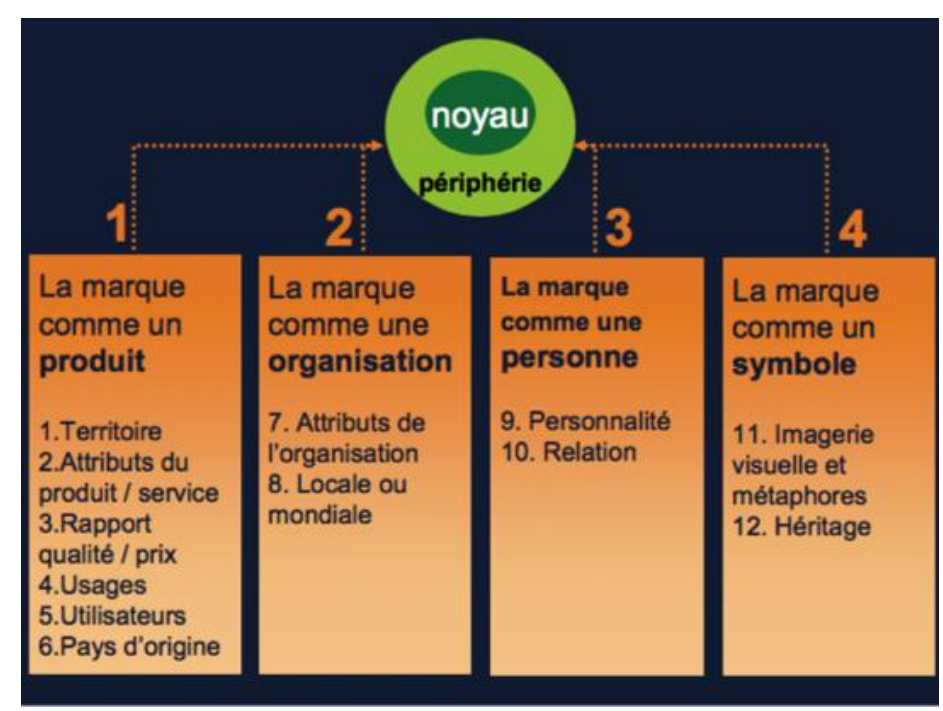

Figure 2 Système d'identité de la marque selon Aaker.

L'identité de marque s'inscrit plus largement dans un projet d'entreprise, une stratégie commerciale et elle traduit en même temps la façon dont l'entreprise, par des signes matériels et immatériels, souhaite se présenter et être représentée.

Une marque englobe un nom, une charte graphique, un logo, une signature, un packaging, un design sonore, visuel, ou olfactif qui lui confèrent une cohérence et une identité propres. L'ensemble de ces éléments s'appuie sur les codes du sensible et du sensoriel. Les packaging, logo, etc. constituent les éléments "visibles» de la marque. Ils s'apparentent à des formes matérielles qui renvoient à des formes symboliques.

Le sociologue Maffesoli (1988) entrevoit l'identité comme un système d'identification, un bricolage de micro récits au sein desquels les identités sociales se lient sur des bases et des affinités connues d'ordre émotionnel. De son côté, A. Semprimi (1999) définit l'identité des marques à partir de trois niveaux : le niveau des valeurs, le niveau narratif et le niveau discursif. A ce propos C. Badulescu (2010: 36 et suivantes) détermine pour la marque Occitane, des valeurs fondamentales d'authenticité, de naturel «narrativisées à travers divers scénarios » mettant en scène le terroir, le savoir-faire et les paysages. Le niveau discursif rend visible la signification de ces valeurs. Cette marque doit tracer « une grammaire sensible » et proposer au public une « parenthèse enchantée ».

Les nouveaux modes et activités de consommation imposent aux marques de choisir de nouvelles stratégies qui leur permettent de s'affirmer par la différence. De même, les consommateurs à l'identité malmenée usent d'objets qui servent aux récits de soi dans un contexte social incertain (Dufour-Baïdouri A., 2013: 16). La marque joue donc un rôle important dans la construction de l'identité individuelle au sein d'une communauté ou d'une tribu et modifie ainsi l'identité collective (B. Heilbrunn).

\section{... A la construction identitaire}

L'identité s'inscrit dans un processus créatif complexe: volonté de distinction, de 
différenciation et besoin de reconnaissance d'un groupe auquel on aimerait appartenir.

Plus que d'une identité établie, il faut évoquer l'idée d'un processus de construction identitaire puisque l'identité se forme et se transforme au sein d'un environnement symbolique et matériel lui-même en perpétuelle mutation.

Ces constructions identitaires sujettes aux évolutions de la société sont alimentées d'une part, par les « consommacteurs » et d'autre part, par les marques qui répondent à leurs attentes.

Analysons précisément quelques exemples significatifs du mécanisme de construction identitaire.

\section{Le processus de construction identitaire des marques commerciales}

Un corpus de publicités va nous permettre d'analyser le concept d'identité d'un point de vue sémiotique et communicationnel. Nous envisagerons également des univers de construction qui révèlent des identités particulières comme le souligne l'expression identité numérique.

La dimension sémiotique de la marque et l'identité se déroulent à travers une " mise en intrigue ». Les manifestations de la marque sont des énoncés qui soulignent la liaison de l'intelligible et du sensible. L'univers de l'intelligible regroupe les composantes abstraites et immatérielles (valeurs, mythologies, promesses, bénéfices, etc.), le sensible recouvre quant à lui les dimensions de la marque perceptibles par les sens (couleurs, matières, odeurs, goût, sonorités, toucher, etc.). La marque considérée souvent comme être humain met en place des éléments d'existence corporelle, sensorielle, relationnelle et sociale et se trouve engagée elle aussi dans des dispositifs d'interaction numériques.

Les voitures ont depuis longtemps constitué des modèles d'analyse révélant des socio-styles de consommateurs. Mais les marques de voiture renferment aussi leur propre identité comme vont l'illustrer les exemples suivants, analysés au prisme des travaux de Kapferer.

Avant de développer l'analyse, présentons rapidement les campagnes publicitaires des trois monospaces qui nous ferons découvrir des ambiances différentes. Nous invitons le lecteur à se reporter aux différents sites sur lesquels nous avons pu repérer les publicités, dont nous donnons les références en notes de bas de pages.

La première analyse se porte sur la marque Renault et la campagne pour le nouvel Espace 3. Les publicités datent de 1994 et correspondent à la série limitée d'un modèle : l'« Espace grand écran » qui va suggérer ce concept d'espace associé au luxe et qui sera largement utilisé dans les publicités qui vont suivre. Les annonces presse ont été photographiées par Paul Wakefield.

En règle générale, l'Espace est placé dans un encart blanc soulignant sa présence discrète dans l'image ou il est délibérément dans un bandeau en dehors de l'image, image qui laisse entrevoir de vastes territoires dans un environnement préservé. Ainsi il s'efface totalement car il n'y a pas de limites à l'espace environnant. Cette campagne offre une liberté de mouvements et de pensée qui laisse place à l'imaginaire des situations convoquées.

La première publicité suggère l'automobile, l'Espace, en ne proposant que les sièges de la voiture au bord d'une plage, dans un environnement assez sauvage et aux couleurs chaudes ${ }^{6}$. L'espace

6 https://pubenstock.wordpress.com/2011/11/20/flashback-renault-espace-1998-agence-publicisquand-lespace-devient-une-garantie-du-luxe/ 
dans tous les sens du terme est au cœur de la publicité alors que la voiture même s'efface au profit d'étendues sans frontières.

La deuxième publicité change d'ambiance mais conserve l'aspect "grandes étendues » avec un château au milieu du désert. Au premier plan dans cet espace de sable, un transat vide et des traces de pas se dirigent vers le château suggèrent la présence humaine. L'imaginaire fonctionne à plein dans ces échanges entre présence et absence, ou tout est suggestion.

Enfin l'environnement aquatique est évoqué dans une autre publicité, un salon confortable est au premier plan pour signaler le confort, comme si on était installé devant un aquarium. Là encore l'espace et le luxe qui y est associé caractérisent la publicité.

Nous reviendrons sur ces publicités en les rapprochant du prisme de Kapferer mais analysons la publicité suivante.

Autre marque, autre stratégie...

En effet, les campagnes publicitaires de la marque Peugeot pour le monospace sont différentes car elles mettent en scène des acteurs (qui sont, comme nous l'avons remarqué, totalement absents dans les publicités précédentes).

La campagne publicitaire Peugeot 806 joue sur l'humour en utilisant les enfants et l'ensemble de la campagne peut se résumer au slogan « la voiture que les enfants conseillent à leurs parents ».

Les enfants sont présentés comme les prescripteurs du véhicule, même si la cible reste bien les adultes. Cette voiture est le symbole d'un esprit «famille» et de parents à l'écoute. La Peugeot 806 participe ainsi de la construction d'une identité de parents attentifs au bien-être et aux désirs de leurs enfants.

Une des publicités reprend phoniquement le nom de la «huit saucisses ». L'enfant tend une assiette remplie de ces huit saucisses, met particulièrement prisé par les enfants de cet âge. Le slogan accompagne l'image «Huit saucisses. La voiture que les enfants conseillent à leurs parents $»^{7}$. L'image est la parfaite traduction ou illustration du nom de la voiture prononcé par un enfant.

Une autre publicité met en scène un enfant en pyjama à rayures et à la mine triste, il est visiblement atteint d'une maladie infantile type "varicelle», des boutons rouges dessinent curieusement sur le corps de l'enfant le nom du produit automobile «806». Les contours d'un thermomètre en bas de l'affiche enferme un message à l'adresse des parents : " Il ne faut surtout pas contrarier un enfant fiévreux $\eta^{8}$. On pourrait ajouter dans la même série, la publicité qui met en scène la voiture et une petite fille bras croisés, au style boudeur, appuyée sur la calandre qui semble vouloir dire « c'est la 806 sinon rien ».

Si l'image diffère à chaque fois, le message est clair : ce sont les enfants qui choisissent et les parents ne doivent pas les contrarier.

Enfin, Citroën :

La campagne pour le Citroën Évasion prend un ton particulier et renvoie à une vague de nostalgie. Le style décalé prend forme en écho au nom du produit (Évasion) et la dimension " amicale », style « soixante-huitard» qui revendique la notion de monospace.

\footnotetext{
${ }^{7}$ http://lemondedesetudes.fr/rhetoriques-dans-la-com-3/\#respond

${ }^{8}$ http://faculty.georgetown.edu/spielmag/docs/semiotique/pubs/peugeot806.jpg
} 
Le slogan «C'est Citroën qui en Premier qui a eu l'idée du monospace » est humoristique et s'analyse en relation avec l'image puisque celle-ci présente un " tube Citroën » aux couleurs noir et blanc, avec l'inscription « Police », c'est-à-dire qu'il s'agit d'un «panier à salade ». Le slogan se poursuit avec le commentaire suivant :

«Le Citroën Évasion possède 5 à 8 places modulables. C'est normal, vous avez toujours eu tellement d'amis » et prolonge l'humour'.

Comparons en nous appuyant sur le prisme de Kapferer les trois identités qui s'affichent sur ces publicités et dont les différences naissent du positionnement, du discours, sur le segment des monospaces.

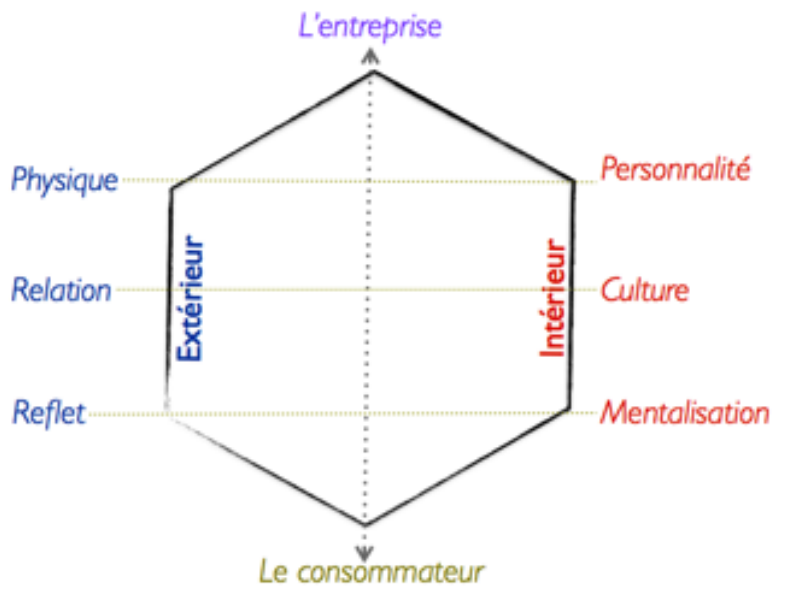

Figure 3 Reprise du prisme de l'identité selon Kapferer

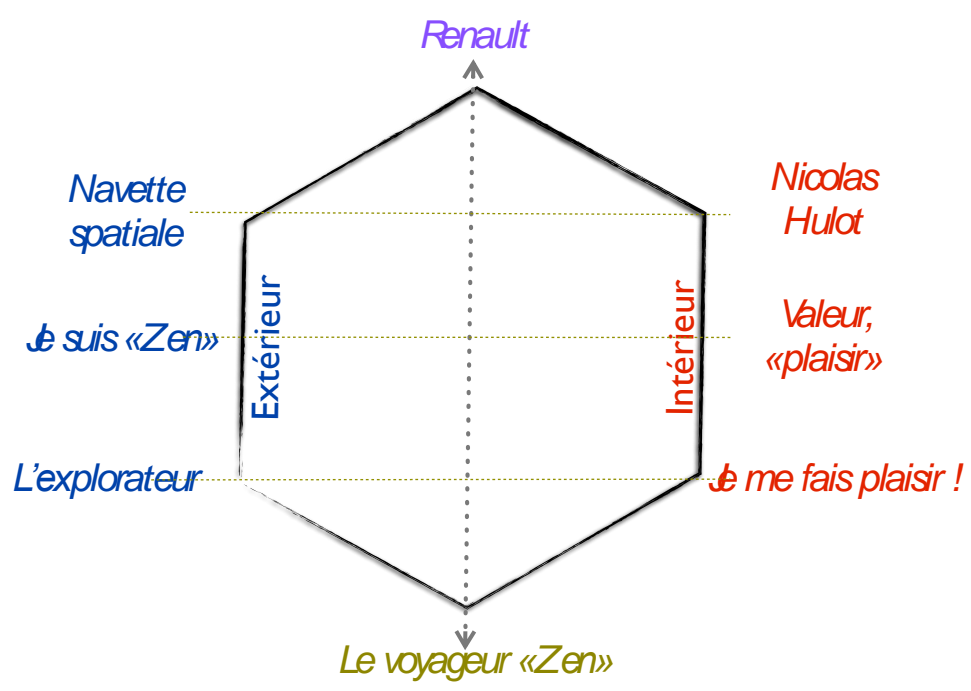

Figure 4 Renault Espace et le prisme de l'identité

${ }^{9}$ http://1.bp.blogspot.com/vHaE48hFZv4/T6wF7AOrQ3I/AAAAAAAAFnw/GRJndobXmL4/s640/1994Citroen.gif 


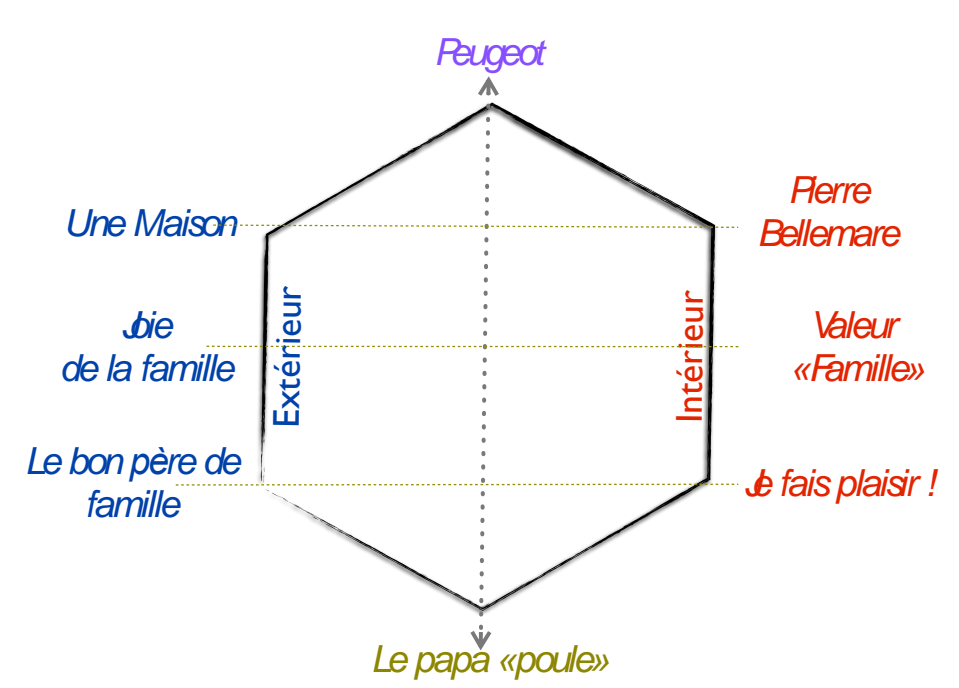

Figure 5 Peugeot 806 et le prisme de l'identité

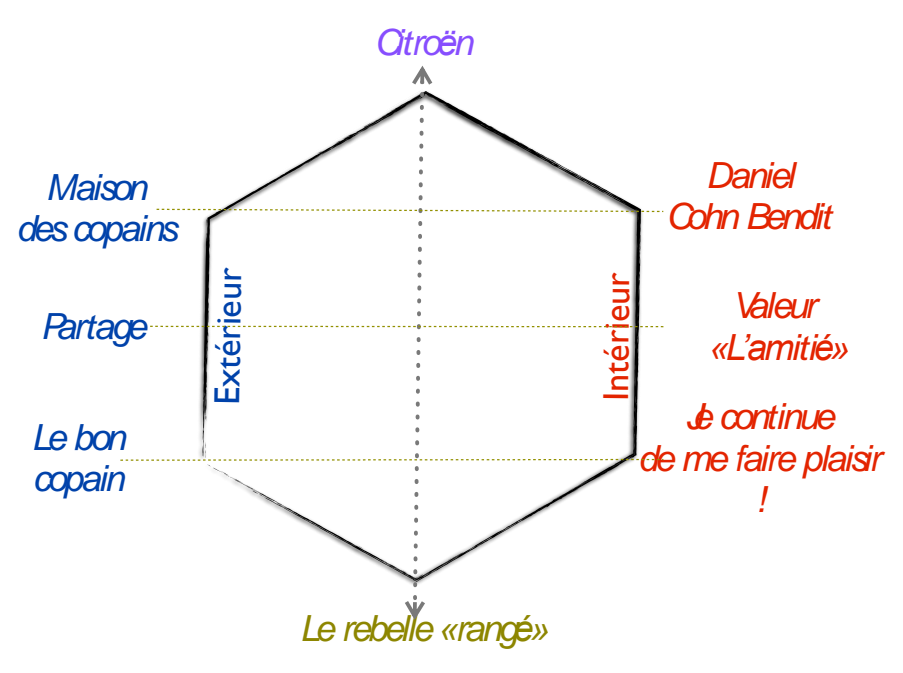

Figure 6 Citroën Évasion et le prisme de l'identité

Les univers déterminés au regard du prisme de Kapferer sont totalement différents.

Pour Renault, l'Espace prend tout son sens. Il correspond bien à ce «grand écran ». Le nom à double sens donne lieu à une exploitation maximale. L'habitacle évoque une navette spatiale qui invite au rêve et l'imaginaire du voyage se développe. Le consommateur est un explorateur et un voyageur Zen qui cherche à se faire plaisir.

Le Peugeot 806 valorise l'univers famille et le plaisir devient une préoccupation collective pour la tribu familiale. L'attention portée aux enfants détermine un consommateur très aimant et surtout «papa poule».

Enfin, le Citroën s'assagit même s'il parle d'Évasion. Avec humour, l'image du car de police est revisitée. On privilégie le contexte de l'amitié, du partage et l'image du rebelle s'est apaisée pour devenir un « rebelle rangé » parce qu'il a mûri, mais conserve le lien avec ses souvenirs.

La notion de plaisir présente dans les trois exemples est abordée de façon différente : entre faire plaisir et se faire plaisir qui exprime des personnalités différentes. 
L'identité de marque peut être en danger dès lors que le positionnement n'est pas clair.

Certains exemples nous le prouvent. Ainsi Nivea a dû modifier sa stratégie. Cette crème centenaire a été créée dans un laboratoire pharmaceutique, dans un contexte de soins. Cependant, afin de se repositionner sur le marché des cosmétiques, elle a évolué de la promesse de soin vers une promesse de beauté. Elle a donc changé ses codes en faisant du co-branding avec Chantal Thomas. Ce positionnement a entraîné l'usage de couleurs plus vives et introduit un aspect glamour teinté de sexualité ${ }^{10}$.

Nivea a dû revenir au positionnement de soin, pour que les consommateurs retrouvent leur identité, tous ayant en mémoire la boîte bleue. Le bénéfice fonctionnel lié à l'aspect hydratation se transforme en bénéfice émotionnel eu égard aux qualités de bien-être, ce qui correspond aux valeurs développées par la société. Le retour au slogan « Nivea et vive la peau » marque en effet cette nouvelle stratégie reprenant la promesse de départ ${ }^{11}$.

Les rites, les symboles et les mythes sont toujours plébiscités par les marques et sont des points d'appui à partir desquels les individus élaborent et construisent des récits sur euxmêmes.

Lavazza, marque italienne célèbre de café s'illustre par une affiche représentant le mythe de Romulus et Remus, mythe fondateur de Rome. Par cette adaptation, la marque vise une certaine tradition au travers de la symbolique du mythe où la louve est substituée par le corps d'une femme sensuelle. Le mythe raconte une histoire et cette histoire contribue a créer l'émotionnel. Le savoir-faire commercial et communicationnel use de la sensualité et de la modernité. Le Colisée, symbole de la ville de Rome sert de décor et ancre un peu plus la notion de tradition. Le produit en retrait s'efface au profit de l'imaginaire de la symbolique du lieu originel et du mythe opéré.

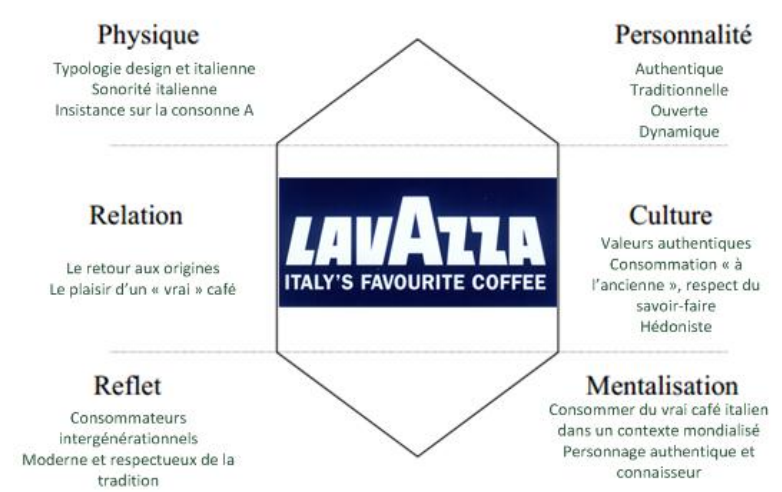

Figure 7 Le prisme de l'identité de Kapferer de Lavazza selon Kotler ${ }^{12}$

\footnotetext{
${ }^{10}$ http://www.webandluxe.com/10/2009/nouvelle-collection-color-passion-par-nivea-et-chantal-thomass/

${ }^{11}$ https://www.bing.com/images/search?view=detailV2\&ccid=weSrtdXn\&id=693E5A4CE8F7614E6FB36B863B2 420E730DC7216\&thid=OIP. weSrtdXnQcswuZNWr2S2yAHaHa\&mediaurl=http\%3a\%2f\%2fimworld.aufeminin.co m\%2fstory\%2f20140729\%2f100-ans-happy-birthday-nivea-

348008_w1000.jpg\&exph $=1000 \&$ expw $=1000 \& q=$ nivea+et+vive + la + peau\&simid $=608030899159566954 \&$ select edIndex $=12 \&$ ajaxhist $=0$

${ }^{12}$ https://demeteretkotler.com/tag/prisme-didentite-de-kapferer/
} 
De même, l'exemple de la Maif paraît intéressant à étudier.

La Maif ${ }^{13}$ est la deuxième compagnie d'assurance préférée des français. Sa notoriété n'est donc plus à faire alors que cette société n'a commencé à faire de la publicité que depuis les années 2000 par besoin de conquérir de nouvelles cibles sur un marché concurrentiel important et de s'inscrire dans un changement de société relevant d'une consommation différente. Depuis l'origine «l'assureur militant» lui a permis de se construire une identité unique dans le monde des assurances. En s'ancrant dès le début dans un discours vrai et transparent, elle a su gagner et toucher les « consommateurs ». La Maif est assureur, c'est son cœur de métier et elle le revendique... Militant indique son engagement dans la société vis-àvis de sa clientèle et elle s'appuie sur des valeurs mutualistes (Liberté, solidarité, démocratie et indépendance) piliers de la doctrine sur laquelle se sont toujours appuyés les mutualistes.

Enfin l'entreprise peut changer d'identité pour montrer qu'elle évolue et qu'elle prend en compte le client.

Subway franchise de restauration rapide fête ses 15 ans et adopte une nouvelle charte graphique, une nouvelle signature. Mais elle cherche avant tout à séduire le consommateur et joue sur la personnalisation proposée au client qui renvoie à la propre identité de ce dernier.

La nouvelle signature "C'est vous le chef» invite le client à être acteur de son choix et la personnalisation donnera un sandwich à son image. Le slogan «Matt\&O présente la version du steak \& cheese » accompagne l'image d'un jeune homme souriant, en tee-shirt sur lequel des bretelles sont visibles, ce qui lui donne un look plutôt sympathique et «cool». La cible des récepteurs est identifiée et correspond à des consommateurs assez actifs sur les réseaux sociaux et «branchés ». La campagne de communication se fera par l'intermédiaire des réseaux sociaux et l'hashtag car le numérique participe à la construction de l'identité d'une marque et à sa large diffusion.

L'identité numérique est un modèle des interactions entre émetteur et récepteur, entre l'individu et la marque, entre les individus eux-mêmes et la gestion de leur identité.

\section{Quand le numérique participe de la construction de l'identité}

Internet, le web 2.0 et les réseaux sociaux ont modifié les cadres sociaux traditionnels, faisant évoluer les rapports des individus entre eux et dans leur relation à la consommation. Ces technologies ont fait naître l'identité numérique. Les individus se sont appropriés ces espaces de communication et d'exposition de soi dans lesquels la liberté d'expression les amène à interagir et à partager sur nombre de sujets dont la consommation. L'évolution des stratégies marketing en est la preuve, les entreprises et les marques doivent maintenant composer avec les communautés de consommateurs virtuels, potentiels ou réels afin de prendre en compte leurs attentes et les fidéliser en développant une relation de confiance et d'engagement mutuel, Parizot (2016).

Ces nouveaux modes de construction identitaire modifient les rapports à la consommation et les marques doivent s'adapter en proposant de nouveaux modes de vie plébiscités par les

\footnotetext{
${ }^{13}$ Une étude très intéressante de l'assureur militant sur :

https://www.ionisbrandculture.com/index.aspx
} 
consommateurs. Récits, narrations permettent aux marques de s'exposer et de répondre aux besoins des individus. La quête de relations, ce nouvel élan du vivre bien et du vivre ensemble par l'intermédiaire de la constitution de tribus ou de communautés, cette recherche d'émotions et de sensations traduite en termes d'expériences incitent les marques à développer des approches du consommateur plus attentives, (Maffesoli, 2012).

Elles fédèrent des communautés de fans et renvoient les consommateurs à la part d'émotionnel qui est en eux, tout en véhiculant leurs propres émotions. Elles partagent de plus en plus avec eux jusqu'à devenir « une œuvre collaborative ${ }^{14}$ » et répondent à la demande de moralisation et d'humanisation des consommateurs en leur proposant des expériences qui les rendent heureux. Les consommateurs se projettent dans la marque et en retour cette dernière s'impose des contraintes. Ainsi, les blogs deviennent de nouveaux espaces de construction de l'identité personnelle mis en place par les consommateurs mais aussi de nouvelles formes de communication des marques elles-mêmes.

\section{Conclusion}

Les marques doivent construire du "sens » car les consommateurs ne sont plus seulement attentifs à la valeur marchande des produits mais aussi à l'identité que ces produits leur confèrent. Ainsi la valeur marchande d'un produit comprend la valeur ajoutée en termes de construction identitaire et sociale de celui qui l'acquiert.

Au-delà de cette construction identitaire personnelle se créent des univers identitaires (le luxe en constitue un). Ainsi la marque Apple confère à son utilisateur une identité de pionner dans la technologie, simultanément épris de simplicité mais non moins attentif au référentiel du luxe.

«[...] La marque Apple cultive chez ses consommateurs le sentiment d'appartenir à un club de rebelles et de gens éclairés guidés par une vérité fondée sur la simplicité [...]. Parvenant à créer étonnement, magie et émotion à partir de produits vendus plus chers que le marché, la marque parvient à jouer sur des tendances de fond de la société, parmi lesquelles l'attrait pour les codes du luxe. De l'emballage aux boutiques en passant par les produits, "Apple a dé-technicisé l'objet par l'esthétique » (Lipovetsky, Le Monde, 27 octobre 2012)» Dufour-Baïdouri A. (2013 : 22).

Les marques apparaissent comme des ressources qui permettent aux consommateurs de puiser dans les mythes, les récits et les symboles pour construire leur propre récit et leur propre identité. Cependant, elles jouissent du retour ou de la collaboration des " consommacteurs » par l'intermédiaire des nouvelles technologies. Ceux-ci relatent leurs expériences et attentes, commentent celles des autres et enrichissent ainsi l'imaginaire collectif.

\section{Bibliographie}

Badot O. (2006), Du sensemaking à l'experiencemaking en marketing, In Les défis du sensemaking en entreprise, p.136-152, Economica.

Badulescu C. 2010 « Le polysensoriel entre approche sémiotique et stratégies marketing» Actes du colloque «Culture et identités dans les communications marketing »78e congrès de l'ACFAS, Centre d'études en communications marketing - UQAM, Université de Montréal, 11 mai 2010. p. 34-42.

\footnotetext{
${ }^{14}$ http://testconso.typepad.com/brandcontent/2012/02/fabriquer-du-sens-.html
} 
Bouchet P., Hillairet D. 2009 Marques de sport. Approches stratégiques et marketing. De Boeck.

Dufour-Baïdouri A. (2013) L'identité numérique. Un levier d'innovation pour les marques? Thèse de doctorat, Université Panthéon-Assas.

Floch, Jean-Marie, Identités visuelles, PUF, coll. Formes sémiotiques, Paris, 1995.

Goffman E. (1974), Les rites d'interaction, Editions de Minuit, Paris.

Heilbrunn B. (2010) «Les marques : créativité ou misère symbolique ». Conférence institut de la mode, Paris [En ligne] http://artindus.hypotheses.org/303

Hetzel P. (2002). Planète conso. Marketing expérientiel et univers de consommation, Editions d'Organisation, Paris.

Kapferer J.-N (1995) Les marques, capital de l'entreprise. $2^{\text {nde }}$ ed 1995. Editions d'Organisation.

Kapferer J.-N. (1988) Maîtriser l'image de l'entreprise : le prisme d'identité. Revue française de Gestion.

Lardellier P, Bryon-Portet C. (2010), «Ego 2.0 ». Quelques considérations théoriques sur l'identité et les relations à l'heure des réseaux, Les cahiers du numérique, $\mathrm{n}^{\circ} 1, \mathrm{p} .13-34$.

Lipovetsky G. (2006), Le bonheur paradoxal, Gallimard, Paris.

Lipovetsky G. (2004), Les temps hypermodernes, Grasset, Paris.

Lipovetsky G. (2003), La société d'hyperconsommation, Le débat, n¹24, mars-avril, p.7498.

Maffesoli M. (1988) Le Temps des tribus. Le déclin de l'individualisme dans les sociétés de masse. Paris, Méridiens-Klincksieck. Rééd. (1991) Le Livre de Poche, Biblio-Essais, 4142.

Maffesoli M. et Perrier B. (2012), L'Homme postmoderne, Ed. CNRS, Paris.

Michon C. et Stern P. (1985) La dynamisation sociale, les Editions d'Organisation, Paris.

Parizot A. (2016) «Marques de confiance et confiance en la marque. Quels signes dans la communication?"In La confiance. Relations, organisations, capital humain, sous la direction de R. Delaye et P. Lardellier. Ed ; EMS, Coll. Entreprise et sacré, pp. 203-226.

Remy-Berger F., (2012), "Gérer l'identité de sa marque. Comment faire bon usage des modèles de Kapferer et AaKer » [En ligne]

http://chaire.marquesetvaleurs.org/sites/default/files/dt/identite_marque.pdf 
